\title{
Solution of dimension abstracted oddity as a function of species, experience, and intelligence'
}

\section{PASCHAL N. STRONG, Jr., ${ }^{2}$ PHILLIP DRASH, ${ }^{3}$ AND MONNIE HEDGES, ${ }^{4}$ TEXAS TECHNOLOGICAL COLLEGE, Lubbock, Texas}

Subjects were divided into two groups, those who had learned one trial, two position, simple oddity, and those who had no formal laboratory experience. Species used were rhesus monkeys, chimpanzees, pre-school humans, 12-year-old humans, young college students and senile adults. No naive monkeys learned Dimension Abstracted Oddity (DAO) while experienced monkeys and chimpanzees did learn. With pre-school children, $I Q$, not experience, was significant in learning $D A O$ while with 12-yearolds and college students the reverse was true. In all species color and form were learned equally easily while height was significantly more difficult to learn.

In a series of recent papers (Strong, 1965; Strong \& Hedges, 1966; Strong, 1966; Strong, 1967) simple oddity learning was investigated using cats, raccoons, monkeys, chimpanzees and humans of various ages.

It was found that normal humans of 12 years or older respond to the oddity principle almost immediately and that a significant proportion of 6 year old children also learn immediately. We wished, therefore, to devise a more difficult problem utilizing the oddity principle and continue our comparative studies using the more difficult problems. To do this, we used a modified version of Bernstein's (1961) Dimension Abstracted Oddity (DAO) problem. METHOD

Subjects. There were two groups of Ss, experienced and naive. Experienced Ss had previously learned one-trial, two-position oddity problems on the WGTA to criterion as described by Strong (1965). Naive Ss had no formal training in oddity learning or on any other problems in our laboratory. Table 1 shows the composition of the experimental groups. The pre-school children were given the Stanford Binet while the 12-year-olds received form EM of the Otis Quick Scoring Intelligence Test.

Human Ss were obtained from sources described in a previous study (St rong, 1966).

Apparatus. The WGTAs used in the simple oddity study were used (Strong, 1965, 1966).

Stimuli and Problems. Stimulus objects were made of wood and were three-dimensional. Four shapes (triangle, square, circle and rectangle), five
Table 1

Summary of Results on DAO Learning for All Groups

\begin{tabular}{lcccc} 
Subject & $\mathrm{N}$ & $\begin{array}{c}\mathrm{N} \\
\text { Learning }\end{array}$ & $\begin{array}{c}\overline{\mathrm{X}} \text { Trials to } \\
\text { Criterion* }\end{array}$ & S.D. \\
\hline Naive Monkeys & 6 & 0 & - & - \\
$\begin{array}{l}\text { Exp. Monkeys } \\
\text { Exp. Chimpanzees }\end{array}$ & 3 & 3 & 1470 & 1680.0 \\
$\begin{array}{l}\text { Naive Children } \\
\quad 40 \text { to 6-11 }\end{array}$ & 20 & 3 & 1310 & 460.0 \\
Exp. Children & & 3 & 760 & 349.0 \\
$\quad 40$ to 6-11 & 20 & 5 & 462 & 482.0 \\
Naive 12-year-olds & 10 & 6 & 495 & 103.6 \\
Exp. 12-year-olds & 10 & 10 & 138 & 45.1 \\
Naive College & 14 & 14 & 212.1 & 100.2 \\
Exp. College & 14 & 14 & 160.8 & 75.0 \\
Naive Senile & 22 & 1 & 270.0 & - \\
Exp. Senile & 7 & 0 & - & - \\
\hline
\end{tabular}

*For thase subjects who learned

colors (red, blue, green, white and yellow) and three sizes (short, medium and tall) were used, making a total of 60 different stimuli. This allows for three basic types of oddity: form, color, and height. A form oddity problem might consist of a small yellow triangle, a medium blue square, and a large red triangle. A typical color oddity problem might consist of a small yellow triangle, a medium blue square, and a large yellow circle. A typical height oddity problem might consist of a short yellow rectangle, a short blue square and a tall red circle. In the height oddity problem, the height differential was always between the short and tall figures. The tall figure was about twice as high as the short figure. From the above examples it can be seen that whenever one dimension is the odd one, the other dimensions are different on each of the three objects.

Six random series of 30 one-trial problems were developed with only one restriction: that of having 10 form, 10 height and 10 color problems in each series. Subjects were given 30 one-trial problems a day until they reached a criterion of $90 \%$ correct for two consecutive days.

Pretraining. Since the experienced Ss had been run on two-position oddity problems they were switched to three-position simple oddity until they were responding at criterion to all positions. They were then introduced to the DAO problem in which the odd stimulus could appear in all three positions.

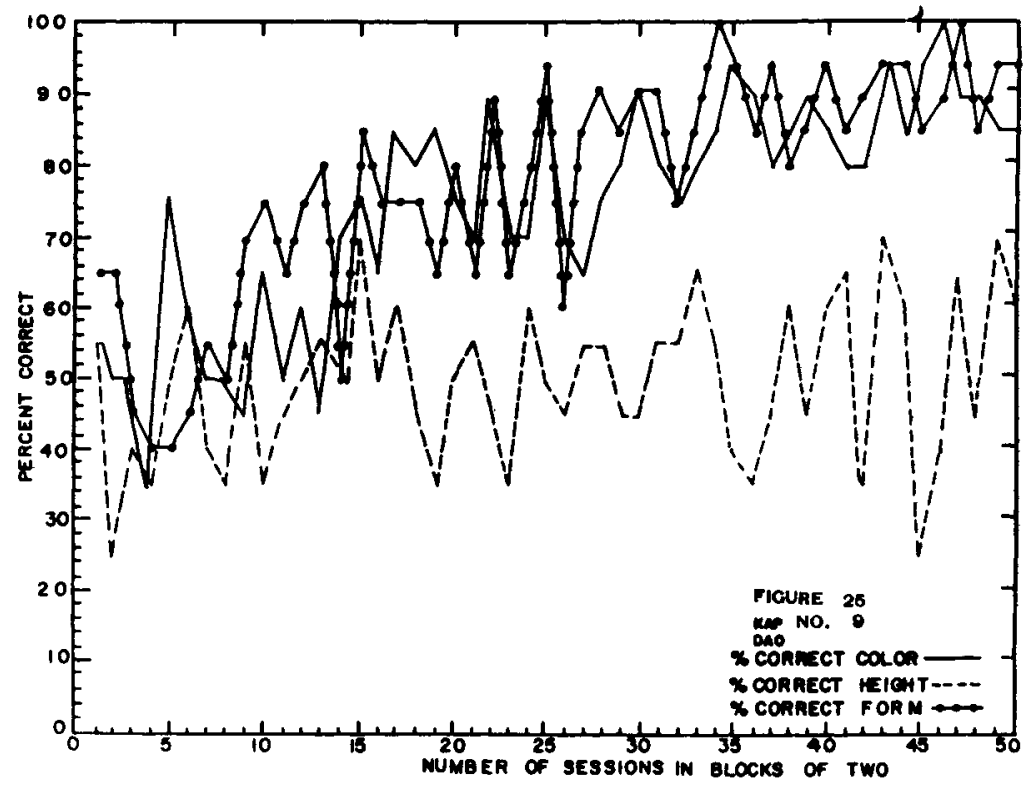

Fig. 1. DAO learning analyzed by dimension for experienced monkey. 


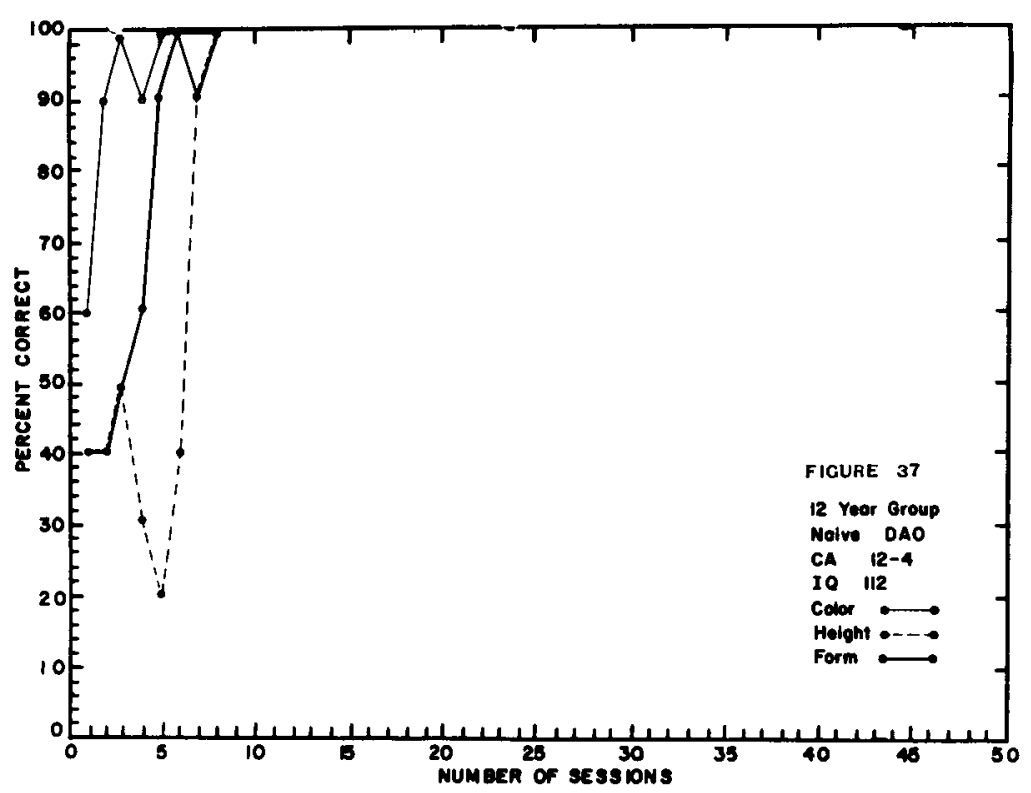

Fig. 2. DAO learning analyzed by dimension for naive 12-year-old male.
The naive Ss were pretrained in exactly the same manner as described by Strong in previous studies $(1965,1966)$.

Reward consisted of raisins and peanuts for the monkeys, candy for the younger humans and pennies for the older humans.

RESULTS

Monkeys and Chimpanzees. Table 1 shows the trials to criterion for all groups. The six naive monkeys were tested for 100 days ( 3000 trials) each and showed no evidence of deviation from chance performance at the end of this time. A breakdown of the three dimensions showed no deviation from chance on any single dimension.

The three experienced monkeys and chimpanzees did learn the DAO problem. Figure 1 shows a learning curve for one monkey analyzed by dimension. For all animals there was no difference in attainment of color and form oddity but height was always the last dimension learned.

Humans. The data for humans is analyzed by age groups. For the pre-school children there was no difference between the naive and experienced Ss either in number learning or trials to criterion. There was, however, a difference in IQ between learners $(\overline{\mathrm{X}}=119.1)$ and nonlearners $(\overline{\mathrm{X}}=98.87)$ at the .01 level $(\mathrm{t}=3.49)$

In the 12 year old group, experience was significant $(t=3.59)$ while IQ was not. The college students showed no significant difference between the experienced and non experienced group $(\mathrm{t}=1.91)$.

Figure 2 shows a typical learning curve for humans. In general, as with lower primates, color and form were learned first with no difference between the two while height was always learned last.

\section{DISCUSSION}

Strong (1965) demonstrated that monkeys who learned one trial oddity on a particular apparatus showed no transfer when given identical problems on a different apparatus. We called this apparatus transfer and further studies with chimpanzees and children indicated that they do show this type of transfer (Strong, 1967). In the present study we are looking at interproblem transfer where apparatus is held constant. Monkeys obviously show this since they cannot solve DAO without simple oddity learning. Furthermore, they show significant savings score since the average trials to criterion for simple oddity was 3952 but for DAO it was only 1470 . It was unfortuante that we could not obtain three more chimpanzees to ascertain if they could have learned DAO without simple oddity experience.
The findings with the children are of interest in light of our past findings. In our pre-school group, IQ, not experience, seemed most important. The youngest mental age to learn DAO was $5-4$. This figure is identical with the youngest MA for our fast learners in simple oddity (those who responded at criterion levels immediately).

All 12-year-olds in our previous study showed an immediate solution to the oddity problem, thus indicating that the concept is firmly incorporated. Consequently experience is more important than mental development and serves as a set producer so that the appropriate response is more likely to be produced. The same is apparently true for the college students since the experience variable just missed significance at the .05 level and was in the same direction as the 12-year-olds.

The consistent findings across species that height is a more difficult dimension than color or form may have been due to one of two causes. Either height is intrinsically more difficult or less obvious to perceive, possibly due to size constancy, or the subjective difference between the two heights was less than between the forms or colors. To test this second possibility, we asked a number of college students to adjust the size of two identical figures so that they appeared as different from each other as a given color pair.

Our results clearly indicated that the size differences we used in this study were of the same magnitude produced by a cross modality match. Therefore we tend to accept the first possibility.

\section{REFERENCES}

BERNSTEIN, I. S. The utilization of visual cues in dimension-abstracted oddity by primates. J. comp. physiol. Psychol, 1961, 54, 243-247.

STRONG, P. N. Learning and transfer of oddity as a function of apparatus and trials per problem. Psychon. Sci, 1965, 3, 19-20.

STRONG, P. N. Comparative studies in simple oddity learning: II. Children, adults and seniles. Psychon. Sci., 1966, 6, 459-460.

STRONG, P. N. Comparative studies in oddity learning: III. Apparatus transfer in chimpanzees and children. Psychon. Sci, 1967, 7, 43.

STRONG, P. N., \& HEDGES, R. M. Comparative studies in simple oddity learning: I. Cats, raccoons, monkeys, and chimpanzees. Psychon. Sci, $1966,5,3-4$.

\section{NOTES}

1. This work was partially supported by Air Force Contract No. 29(600)-2939.

2. Now at the University of South Florida.

3. Now at Johns Hopkins Medical School.

4. Now at Eastern Carolina University. 